\section{EDAX Inc.}

\section{Company Description}

Founded in 1962, EDAX is the world's largest supplier of EDS, EBSD, and WDS systems for electron microscopy applications and Micro-XRF systems, providing technical and performance leading solutions for materials characterization. EDAX's seamlessly integrated systems provide a powerful combination of elemental information from EDS and structural information from EBSD offered in the Pegasus system, while the Neptune offers advanced X-ray microanalysis by combining EDS and WDS. The EDAX Trident system offers a unique combination of EDS, EBSD, and WDS in one analytical tool that combines the latest technological advancements for all three techniques.

\section{Advancing Technology}

EDAX continues to lead the way in EDS technology with the new TEAM ${ }^{\mathrm{sm}}$ EDS Analysis System. All the knowledge and experience of an EDS expert is built into this advanced system. Smart Features provide analytical intelligence and allow the user to obtain quality and reliable results for a wide range of applications. The monitoring and operating conditions of the system are available with Smart Track. Optimal set-up of the image collection occurs with Smart Acquisition. Available to the user is guidance and dynamic adjustment of the map collection with Smart Phase Mapping. TEAM EDS offers the most accurate peak identification and quantification in the industry with EDAX's revolutionary EXpert ID. Reports are generated in a single button click with Smart Data Review. No other system on the market offers this type of built-in analytical intelligence. This advanced system was designed to change the way the scientist does analysis forever. The Apollo Silicon Drift Detector series (EDS), Hikari camera (EBSD), and TEXS HP (WDS) provide today's scientist with the ultimate materials characterization solution, offering vastly improved speed and accuracy.

The Orbis micro-XRF inorganic elemental analyzer system sets a new standard in analytical flexibility. The Orbis incorporates a unique motorized turret for coaxial sample view and X-ray analysis. Primary beam filters can be used with $\mathrm{X}$-ray optics for tailored micro to millimeter spot elemental analyses. Orbis micro-XRF measurements are non-destructive, require minimal sample preparation and offer improved sensitivity over SEM/EDS.

\section{Applications and Markets Served}

EDAX instrumentation for elemental and structural analysis is found in a broad spectrum of industrial, academic, and government applications from the quality control department to the most advanced research and development laboratory. Typical markets served include semiconductor and microelectronics, academic and industrial R\&D laboratories, ROHS/WEEE, renewable energy, pharmaceuticals, mining, security, forensics, catalysts, petrochemicals, metallurgy, and manufacturing operations.

EDAX raises the standard as the technical innovator with high performance and functionality that gives you Confidence in Your Results.

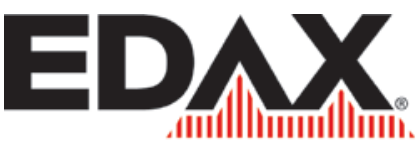

advanced microanalysis solutions

AMETEK

MATERIALS ANALYSIS DIVISION

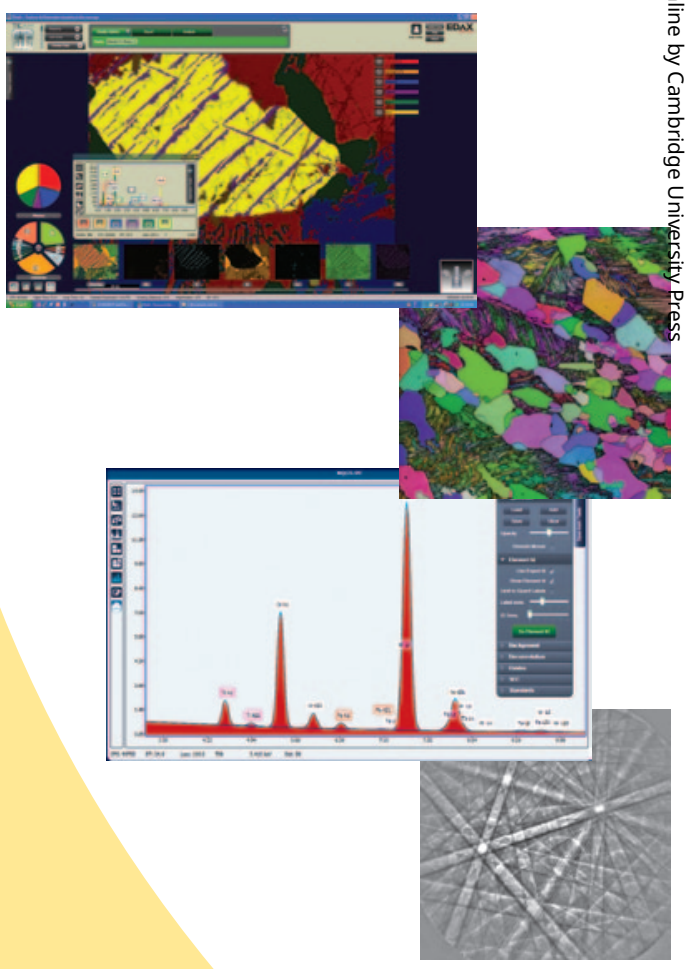

\section{How to find us}

With major facilities worldwide, EDAX is well positioned to support its vast and growing network of satisfied customers. EDAX headquarters is located in Mahwah, NJ, housing sales, technical support and manufacturing operations. EDAX is committed to providing the best possible support for our customers worldwide with sales, service and applications support offices located in Japan, China, Singapore, the Netherlands, Germany, UK, and the United States.

EDAX Inc.

91 McKee Drive

Mahwah, NJ 07430

Tel: 1-201-529-4880

Fax: 1-501-529-3156

Email:info.edax@ametek.com

Web: www.EDAX.com 